\title{
Département du Gers
}

\section{Catherine Petit et Pierre Sillières}

\section{OpenEdition \\ Journals}

Édition électronique

URL : http://journals.openedition.org/adlfi/10738

ISSN : 2114-0502

Éditeur

Ministère de la culture

Référence électronique

Catherine Petit et Pierre Sillières, « Département du Gers », ADLFI. Archéologie de la France -

Informations [En ligne], Midi-Pyrénées, mis en ligne le 01 mars 1997, consulté le 19 avril 2019. URL

http://journals.openedition.org/adlfi/10738

Ce document a été généré automatiquement le 19 avril 2019

(c) Ministère de la Culture et de la Communication, CNRS 


\title{
Département du Gers
}

\author{
Catherine Petit et Pierre Sillières
}

Date de l'opération : 1988 - 1991 (PR) ; 1988 - 1991 (PA)

Inventeur(s) : Petit Catherine ; Sillières Pierre

1 Depuis 1986, une opération de couverture aérienne systématique du département est mise en œuvre (Gallia Informations, 1989: 172) et s'appuie en grande partie sur la documentation existante, sur l'étude des sources et de la toponymie mais aussi sur des prospections au sol effectuées par des étudiants de l'université de Toulouse - Le-Mirail, sous la direction de P. Sillières (CNRS) et de C. Petit.

Après avoir pris pour base de travailles sites déjà répertoriés comme, par exemple, celui de la Sioutat à Roquelaure (villa ? et gisement du $\mathrm{I}^{\mathrm{er}}$ s. avant J.-C., Gallia, 1972 : 497) ou de Saint-Clar à La Bénazide d'Ayrem (Gallia, 1970 : 418) dont le plan au sol et la chronologie ont pu être complétés, la presque totalité des informations recueillies proviennent de gisements inédits.

Pour la période préhistorique, une seule station de plein air est signalée à l'Isle-Bouzon, La Boubée (outillage en silex du Paléolithique ?), alors que pour l'Antiquité, les villoe galloromaines sont nombreuses : Auch, Lagoudagne (mobilier céramique compris entre le $\mathrm{I}^{\mathrm{er}} \mathrm{s}$. et le IV ${ }^{\mathrm{e}}$ s. après J.-C.), (Fig. $\mathrm{n}^{\circ} 1$ : Auch (Gers). Lagoudagne. Villa et dépendances) ; Avezan, Le Cauze ( $\mathrm{I}^{\mathrm{er}} \mathrm{s}$. au IV $\mathrm{I}^{\mathrm{e}} \mathrm{s}$. après J.-C.) ; Bivès, Chirquoy (villa autour de la chapelle de NotreDame-des-Neiges) ; Castelnau-d'Arbieu, Orino, (découverte d'une tête funéraire (?) féminine en marbre) ; Lectoure, Cujon, avec la mise en évidence de l'aile nord de la villa ( Gallia Informations, 1989 : 172) ; Pergain-Taillac, Saint-Martin ; Roquelaure, Garmazan (villa à cour centrale et à galerie de façade), (Fig. $n^{\circ} 2$ : Roquelaure (Gers). Garmazan. Villa à cour centrale et à galerie de façade); Toujet, Lapalue.

4 La parsurbana est parfois associée à des fermes dépendant de la parsrustica comme à SaintClar, Ayrem, Labarthe, Merlane, Le Moulin d'Ayrem, alors que des bâtiments agricoles isolés se reconnaissent à Cadeilhan, La Saudroue et à Saint-Clar, Arruats.

Des établissements gallo-romains, difficilement interprétables en l'état, sont également apparus à Augnax, La Bordeneuve; Isle-Bouzon, En Bordes, Milord, Montagnac, au 
Village ; Lectoure, Flageoulet (structure carrée aux angles arrondis) ; Lacroux ; Marsolan, Doat (structure rectangulaire) et Garbeau (quatre ensembles orientés différemment et dépendant, d'après le prospecteur, de la partie agricole d'un établissement rural. C'est de cet endroit que proviendrait une inscription DIVAE FAVSTINAE découverte anciennement).

6 Quelques éléments lapidaires isolés ont encore été repérés à Castelnau-d'Arbieu, Orino (tête funéraire (?) féminine en marbre), LasTucolles (fronton triangulaire avec acrotères); Saint-Clar, Ayrem (buste de divinité (?) en calcaire); Sempesserre, Cazeneuve (tête féminine en marbre), Clarissens (personnage debout, vêtu de sa tunique). Il convient encore de signaler, pour cette période, la mise en évidence de plusieurs tronçons de la voie romaine Toulouse (Tolosa) - Lectoure (Lactora)/ (Tournecoupe, Enjoinques et Avezan / Saint-Clar, LesBoyes / Au Petit Frans; Auch (Elimberris, civitas Auscorum) Lectoure (Lactora) (Auch, Ritou); Auch - Eauze (Elusa) (Eauze, Martin et Biran, La Caussade).

7 Concernant l'époque médiévale, les résultats sont moindres mais plutôt spectaculaires: Montestruc-sur-Gers, La Bordeneuve (structures creuses composées de deux fossés de forme subcirculaire, associés à d'autres structures indéterminées, mais aussi, sur le terrain, à une faible levée de terre), (Fig. n³ 3 : Montestruc-sur-Gers. La Bordeneuve. Double enclos subcirculaire associé à d'autres structures creuses indéterminées); groupes de silos à Roquelaure, En Sajas; Saint-Georges, En Degans; Sempesserre, à La Sebette / à Campagnan (deux ensembles de fosses/silos ? - vingt-neuf, d'une part, et une centaine, d'autre part - distants de $400 \mathrm{~m}$, dont certains paraissent alignés et qui ont livré en prospection de surface, du matériel daté entre le $\mathrm{X}^{\mathrm{e}} \mathrm{s}$. et le XII ${ }^{\mathrm{e}}$.) (Fig. $\mathrm{n}^{\circ} 4$ : Sempesserre (Gers). La Sebette/ A Camparan. Ensemble de fosses-silos).

8 Une route mentionnée sur une carte $\mathrm{du} \mathrm{XVI}{ }^{\mathrm{e}}$ s.et disparue sur le cadastre napoléonien a été localisée à Saint-Georges, au Couloumé / au Barthas et une motte castrale à Sempesserre, Lamothe, ainsi que des vestiges d'époque moderne à Saint-Clar, Lantra et En Calaves.

9 Parmi les observations dont la détermination morphologique et chronologique reste imprécise, on peut reconnaître des structures rectangulaires à Saint-Antonin, Las Paguères et Saint-Clar, Lantras, associées à un parcellaire fossile. Il y a aussi des structures circulaires à Lectoure, Noulieu et La Grande Pièce comme à Avenzac, au Bousqué; des tronçons de fossés curvilignes à Ordan-Larroque, Entrouet et SaintAntonin, La Gimbrède.

10 S'il reste encore beaucoup à faire, l'importance des données acquises jusqu'à ce jour et qui, pour la plus grande part, étaient inédites, justifie pleinement la poursuite de cette opération exemplaire pour la région Midi-Pyrénées. Au-delà de ce constat, il conviendra de réfléchir sur la perspective d'une étude d'ensemble portant sur l'occupation du sol et sur une réflexion touchant le domaine de la voirie antique. 
ANNEXES

Fig. $\mathrm{n}^{\circ} 1$ : Auch (Gers). Lagoudagne. Villa et dépendances

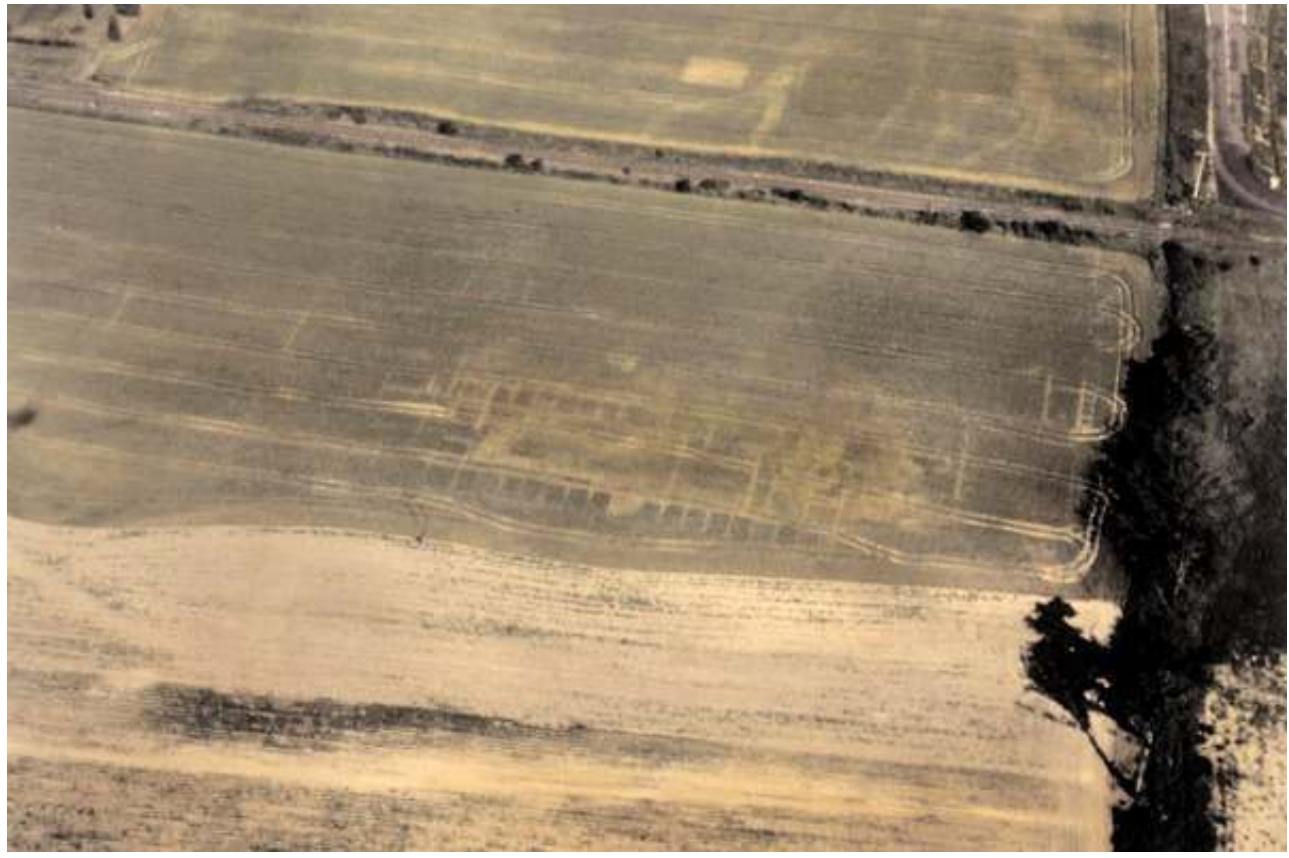

Auteur(s) : Petit, Catherine. Crédits : GI, 1997 - CNRS Editions, 1998 (1997)

Fig. $n^{\circ} 2$ : Roquelaure (Gers). Garmazan. Villa à cour centrale et à galerie de façade

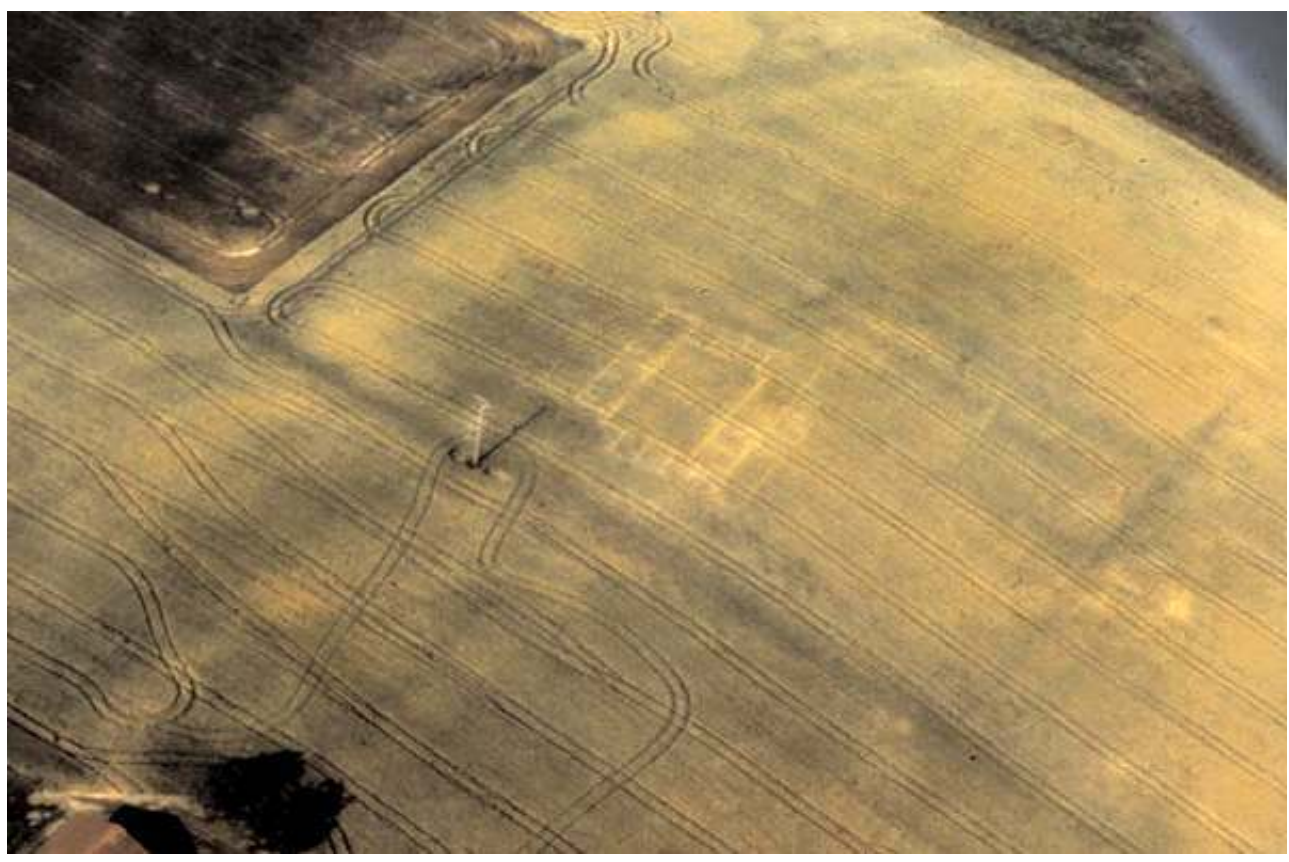

Auteur(s) : Petit, Catherine. Crédits : GI, 1997 - CNRS Editions, 1998 (1997) 
Fig. $n^{\circ} 3$ : Montestruc-sur-Gers. La Bordeneuve. Double enclos subcirculaire associé à d'autres structures creuses indéterminées

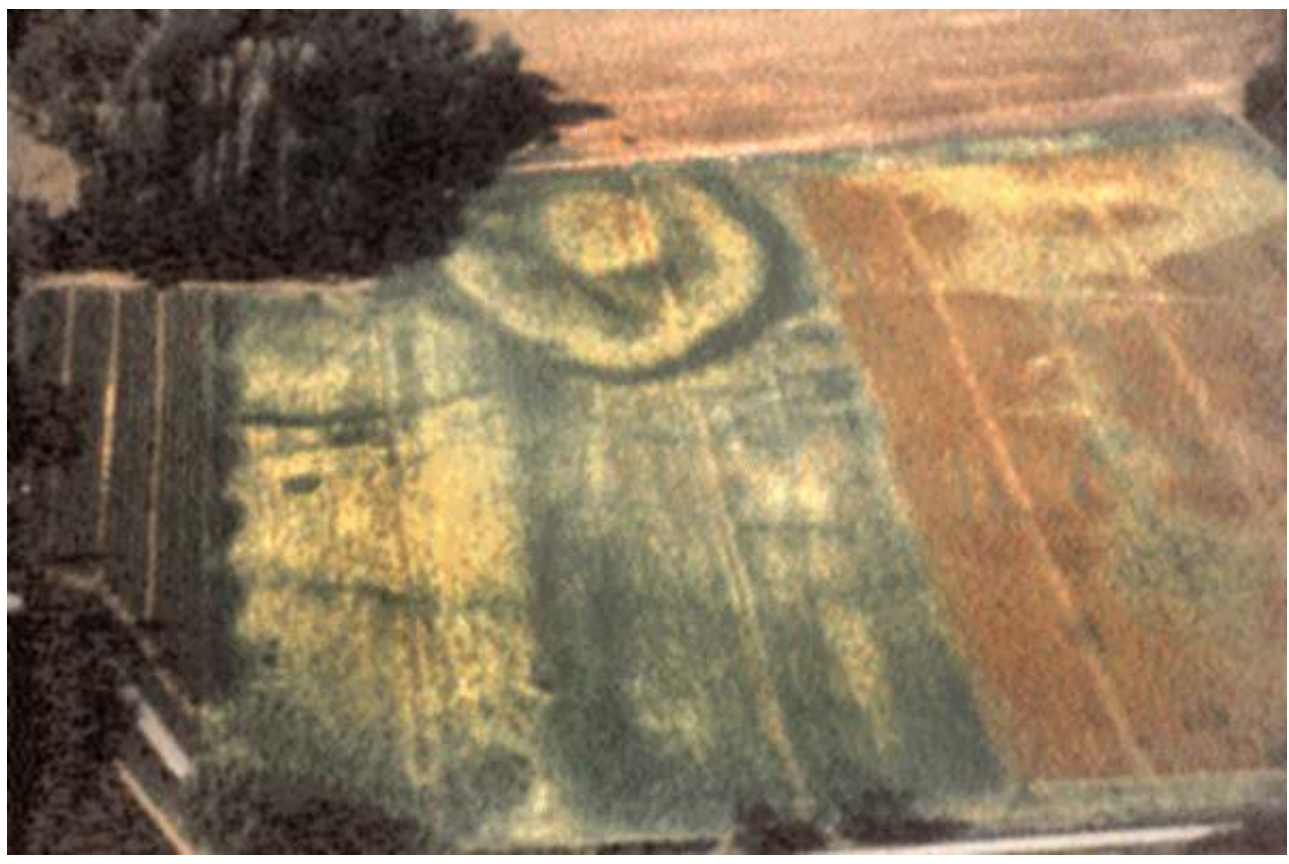

Auteur(s) : Sillières, Pierre. Crédits : GI, 1997 - CNRS Editions, 1998 (1997)

\section{Fig. $\mathrm{n}^{\circ} 4$ : Sempesserre (Gers). La Sebette/ A Camparan. Ensemble de fosses-silos}

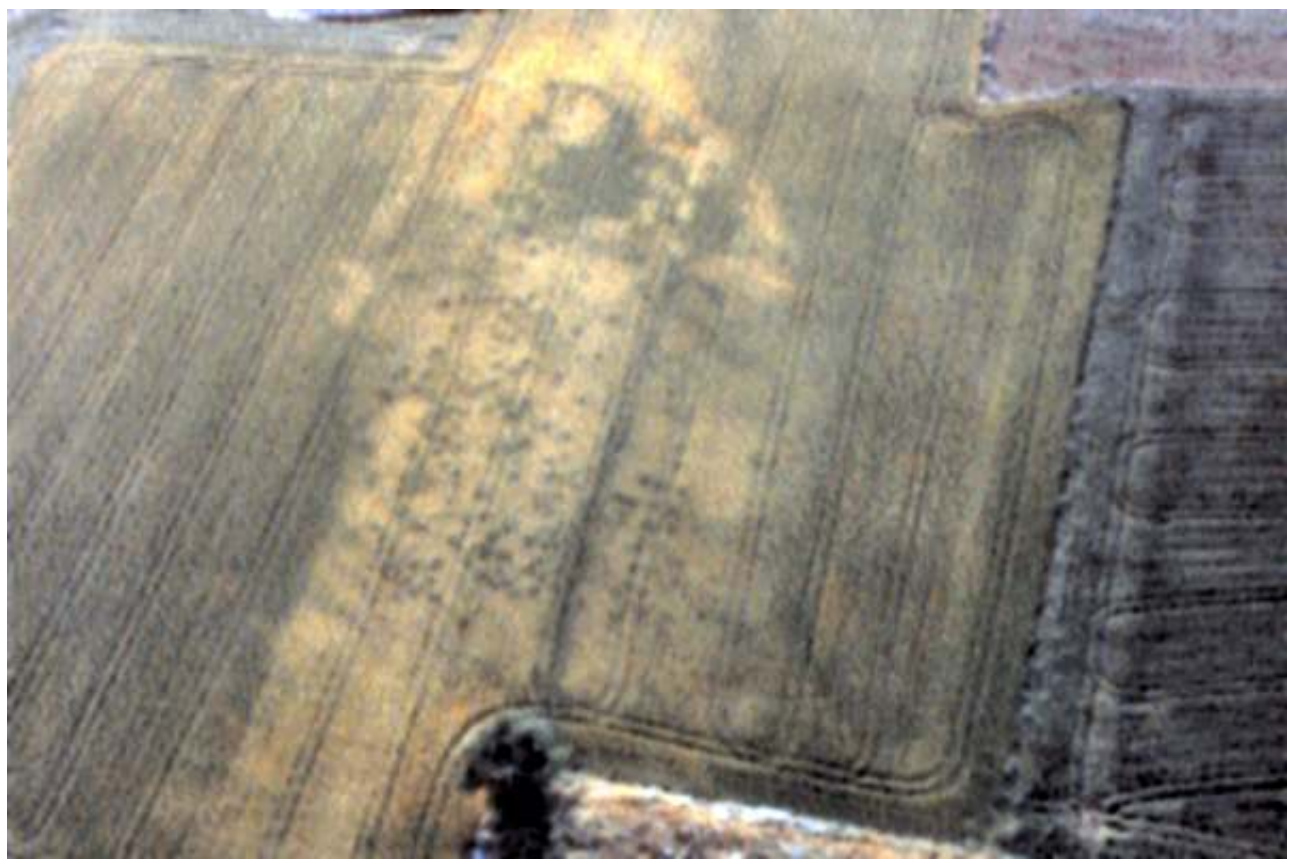

Auteur(s) : Petit, Catherine. Crédits : GI, 1997 - CNRS editions, 1998 (1997) 
INDEX

Index chronologique : Antiquité romaine, Moyen Âge, Paléolithique, Temps Modernes operation Prospection (PR), Prospection aérienne (PA)

Index géographique : Midi-Pyrénées, Gers (32), Auch, Isle-Bouzon (32158), Avezan, Roquelaure, Montestruc-sur-Gers, Sempesserre 